\title{
Immunological Detection of Small Organic Molecules in the Presence of Perchlorates: Relevance to the Life Marker Chip and Life Detection on Mars
}

\author{
Catherine S. Rix, Mark R. Sims, ${ }^{2}$ and David C. Cullen ${ }^{1}$
}

\begin{abstract}
The proposed ExoMars mission, due to launch in 2018, aims to look for evidence of extant and extinct life in martian rocks and regolith. Previous attempts to detect organic molecules of biological or abiotic origin on Mars have been unsuccessful, which may be attributable to destruction of these molecules by perchlorate salts during pyrolysis sample extraction techniques. Organic molecules can also be extracted and measured with solventbased systems. The ExoMars payload includes the Life Marker Chip (LMC) instrument, capable of detecting biomarker molecules of extant and extinct Earth-like life in liquid extracts of martian samples with an antibody microarray assay. The aim of the work reported here was to investigate whether the presence of perchlorate salts, at levels similar to those at the NASA Phoenix landing site, would compromise the LMC extraction and detection method. To test this, we implemented an LMC-representative sample extraction process with an LMCrepresentative antibody assay and used these to extract and analyze a model sample that consisted of a Mars analog sample matrix (JSC Mars-1) spiked with a representative organic molecular target (pyrene, an example of abiotic meteoritic infall targets) in the presence of perchlorate salts. We found no significant change in immunoassay function when using pyrene standards with added perchlorate salts. When model samples spiked with perchlorate salts were subjected to an LMC-representative liquid extraction, immunoassays functioned in a liquid extract and detected extracted pyrene. For the same model sample matrix without perchlorate salts, we observed anomalous assay signals that coincided with yellow coloration of the extracts. This unexpected observation is being studied further. This initial study indicates that the presence of perchlorate salts, at levels similar to those detected at the NASA Phoenix landing site, is unlikely to prevent the LMC from extracting and detecting organic molecules from martian samples. Key Words: Mars-Perchlorate salts-ExoMars-Life Marker Chip-Immunoassay—Life detection-Oxidants. Astrobiology 11, 839-846.
\end{abstract}

\section{Introduction}

I N 2008, the Wet Chemistry Laboratory on NASA's Phoenix lander detected $0.4-0.6 \mathrm{wt} \%$ perchlorate salts $\left(\mathrm{ClO}_{4}^{-}\right)$in regolith samples collected from the martian surface and immediate subsurface at high latitudes (Hecht et al., 2009; Kounaves et al., 2010). This discovery has led to discussion of the implications for life on Mars (Kerr, 2010; Stoker et al., 2010), including the speculation that extant martian life might utilize a water-perchlorate mixture to overcome the subzero conditions on Mars today, based upon an earlier suggestion of the use of hydrogen peroxide water mixtures (Houtkooper and Schulze-Makuch, 2007). The discovery of perchlorates has also led to re-evaluation of the results from the Viking biology experiments of the 1970s (Navarro-González et al., 2010) and has offered an explanation for the failure of past analyses to detect any organic molecules (biologically produced or otherwise) on Mars despite their continuing delivery to the surface in meteoritic material (ten Kate, 2010) independently of any past or present-day biology on the planet.

A number of different geologies and geochemical reservoirs are present on Mars that may contain iron-, magnesium-, calcium-, potassium-, and sodium-based perchlorates, depending on the geological and hence geochemical history and conditions at a given location. Two types of perchlorates have been investigated here, namely, calcium and magnesium, for reasons described in the Experiment Design section of this paper.

Perchlorate salts are oxidizing, but metastable, unless initiated by mechanical action, heating, or electric shock (Shriver

\footnotetext{
${ }^{1}$ Cranfield Health, Cranfield University, Cranfield, United Kingdom.

${ }^{2}$ Space Research Centre, Department of Physics and Astronomy, University of Leicester, Leicester, United Kingdom.
} 
and Atkins, 1999); therefore, they are not expected to destroy organic molecules under martian conditions. NavarroGonzález et al. (2010), however, demonstrated that when organic carbon containing Mars-like soils from the Atacama Desert is mixed with $1 \mathrm{wt} \%$ magnesium perchlorate and heated, the organics present are decomposed. All previous attempts to detect organic molecules on Mars, for example by the gas chromatograph-mass spectrometer (GC-MS) instrument on the Viking landers and Phoenix's Thermal and Evolved Gas Analyzer (TEGA), have relied on pyrolysis to volatize organics present in collected samples. In the Viking experiments, samples were heated to $200^{\circ} \mathrm{C}, 350^{\circ} \mathrm{C}$, and $500^{\circ} \mathrm{C}$ (Anderson et al., 1972), and TEGA progressively heated samples up to a temperature of $1000^{\circ} \mathrm{C}$ (Hoffman et al., 2008). Heating to these temperatures in the presence of perchlorates may have destroyed the very molecules that the Viking GC-MS and TEGA were trying to detect.

The proposed ExoMars Rover (Vago and Kminek, 2007) is equipped with a drill capable of collecting rock cores from a maximum depth of $2 \mathrm{~m}$ and will carry a payload that currently includes two instruments capable of detecting trace levels of organic molecules: Mars Organic Molecule Analyser (MOMA) and the Life Marker Chip (LMC). Additionally, a Raman spectrometer instrument included in the payload will be capable of organics detection if organics are present at suitable levels.

Mars Organic Molecule Analyser is a compact, low-power ion trap mass spectrometer with the capability to extract organics from martian rock and regolith samples by two different methods: pyrolysis and laser desorption (EvansNguyen et al., 2008). Extraction by pyrolysis will involve evaporation of volatile organics in the martian sample by heating to high temperatures followed by injection into a GC column with an electron ionization source. Exposure of the martian sample to high temperatures could result in perchlorate-mediated degradation, although recently it has been proposed to integrate the use of derivitization reagents to increase the volatility of some organics in order to reduce the required temperature (Eigenbrode et al., 2011). In extraction by laser desorption, the regolith sample will be pulsed with a high-power UV laser to liberate ions (ESA, 2011); it is unknown whether this technique will be affected by the presence of perchlorates.

The aim is for the LMC, which is under development by a UK-led consortium, to analyze martian samples for a predefined set of up to 25 organic molecules by using a multiplexed microarray inhibition format immunoassay. The current instrument is a variation of the original concept published earlier (Sims et al., 2005) and uses fluorescence detection, while the earlier version used refractive index changes during binding. Target molecules for the LMC include biologically produced markers of extant Earth-like life, the digenesis products of these molecules as markers of extinct life, and abiotically produced organic molecules such as polyaromatic hydrocarbons that result from meteoritic infall (Parnell et al., 2007).

Briefly, the current design of the LMC consists of four "single use" modules that can each extract and analyze a single, approximately $1 \mathrm{~g}$ sample of martian regolith or crushed rock. On Mars, the drill and associated sample processing and distribution system on the ExoMars Rover will deliver a $1 \mathrm{~g}$ sample of regolith or crushed rock to one module of the LMC. The sample will be subject to liquid extraction with use of an aqueous/miscible organic solvent/surfactant mixture (Court et al., 2010) together with the application of ultrasonic energy to extract and solubilize polar and apolar organic molecules. The extraction solvent has been developed as a compromise between efficient extraction of target organic molecules and immunoassay performance. The liquid extract will then be pumped into the analysis system of the LMC, which consists of microfluidic channels that allow the implementation of a multiplexed inhibition immunoassay in a format similar to a lateral flow immunoassay (PosthumaTrumpie et al., 2009). The key aspects of the immunoassay within the microfluidic channel are two chambers: one chamber contains a "freeze-dried cocktail" of immunoassay reagents that include fluorescently labeled antibodies that dissolve into the liquid extract, and a second chamber contains an immobilized microarray of protein conjugates of the target molecules. Final readout of the immunoassay signal is achieved via imaging of the presence of the fluorescent labels on the microarray with a CCD-based light-detection system. Therefore, if target molecules are present in the extract from the martian sample, they will bind to their corresponding fluorescently labeled antibodies, as these dissolve into the liquid extract, and will subsequently inhibit the binding of the fluorescently labeled antibodies to the corresponding spots of protein conjugates of the target molecules within the microarray. The end result is a fluorescent signal that decreases with increasing target molecule concentration.

The LMC's use of a low-temperature $\left(>0^{\circ} \mathrm{C}, \ll+100^{\circ} \mathrm{C}\right.$, nominally $+20^{\circ} \mathrm{C}$ ) liquid extraction may offer an advantage over other instruments because perchlorate salts are not expected to act as oxidants under these conditions. However, perchlorates are highly water soluble and therefore expected to dissolve in the LMC extraction solvent and be carried through into the LMC analysis system where the perchlorates could interact with immunoassay reagents via changes in parameters such as $\mathrm{pH}$ and ionic strength. Within the immunoassay literature, there appear to be no examples that demonstrate detection of small organic molecules by immunoassay in the presence of perchlorate salts, although there is one recently published study that describes no significant effect of $50 \mathrm{mM}$ perchlorate on the antibody-antigen reaction by sandwich-type antibody microarray fluorescent immunoassays (Parro et al., 2011). Therefore a study is required to establish (a) whether inhibition immunoassays can function effectively in the presence of a Mars-relevant concentration of perchlorate and (b) whether inhibition immunoassays can function effectively in an extract from a sample spiked with a Mars-relevant concentration of perchlorate. The study reported here addresses these points in the immediate context of the LMC and is designed primarily as a "de-risking" study to identify major problems that would impact upon the LMC development schedule. This is a timely study given the current development schedule of the LMC and more broadly the ExoMars mission.

\section{Materials and Methods}

\subsection{Chemicals and biochemicals}

All reagents were purchased from Sigma-Aldrich (Poole, UK) unless otherwise stated. 4-(Benzo[a]pyren-6-yl)butanoic acid was procured from Sigma-Aldrich Fine Chemicals 
Division (Poole, UK) as a custom synthesis and conjugated to bovine serum albumin (BSA) with standard EDC/NHS coupling chemistry. Mouse anti-benzo[a]pyrene clone 22F12 was supplied by Prof. Dr. D. Knopp, (Technical University of Munich, Germany). Goat anti-mouse IgG Fc biotin was purchased from QED Biosciences (San Diego, USA), and streptavidin-horseradish peroxidase (HRP) was purchased from Invitrogen (Paisley, UK). Methanol was purchased from Fisher Scientific (Loughborough, UK) and was HPLC grade. All water used was $18 \Omega \cdot \mathrm{cm}$ and was purified with a Direct Q UV-3 water purification system (Millipore, Massachusetts, USA). Calcium perchlorate tetrahydrate (product code 401420) and magnesium perchlorate hexahydrate (product code 309303) were purchased from Sigma-Aldrich.

\subsection{Spiking of martian regolith simulant with pyrene and perchlorates}

The martian regolith simulant JSC Mars-1 (used as supplied) was spiked with pyrene in methanol to a final concentration of $500 \mu \mathrm{g}$ pyrene/g simulant regolith. This level was chosen in the combined context of the expected extraction efficiency, the known sensitivity of the model immunoassay used, and an additional factor to produce extracts that gave $100 \%$ immunoassay inhibition in the control samples. It is noted that this results in a higher level of pyrene than could be expected on Mars. To achieve this, $500 \mu \mathrm{L}$ of $1 \mathrm{mg} / \mathrm{mL}$ pyrene in methanol was added to $1 \mathrm{~g}$ of JSC Mars- 1 in a $7 \mathrm{~mL}$ glass tube, mixed by vortexing, and allowed to stand at room temperature for approximately $30 \mathrm{~min}$. The JSC Mars-1, which consisted of a free-flowing granular matrix when dry, formed into clumps when wetted with solvent. After standing, methanol was removed by evaporation; vials were loosely sealed and placed in an oven set at a temperature of $37^{\circ} \mathrm{C}$ overnight. After approximately $18 \mathrm{~h}$, the JSC Mars-1 had reverted to a free-flowing granular matrix. Control samples were spiked with methanol containing no pyrene and mixed and dried in the same way as the pyrenespiked samples.

After spiking with pyrene, JSC Mars-1 was spiked with $1 \mathrm{wt} \%$ perchlorate anion by addition of solid calcium perchlorate tetrahydrate or magnesium perchlorate hexahydrate to the dry simulant regolith. Fifteen and seven-tenths milligrams of calcium perchlorate or $16.7 \mathrm{mg}$ of magnesium perchlorate (both fine crystalline powders, used as supplied) were weighed into $7 \mathrm{~mL}$ glass vials, and $1 \mathrm{~g}$ of dried pyrenespiked JSC Mars-1 was transferred into each vial and mixed well by inversion and by vortex mixer.

\subsection{Extraction of spiked JSC Mars-1}

Three milliliters of $20 / 80 \%(\mathrm{v} / \mathrm{v})$ methanol/water with $1.5 \mathrm{~g} / \mathrm{L}$ polysorbate 80 was added to $1 \mathrm{~g}$ spiked JSC Mars-1 in a glass test tube and sonicated for $20 \mathrm{~min}$ with a Sonotrode Ultrasonic UP100H probe and UP50H ultrasonic processor set at $40 \%$ amplitude (Hielscher Ultrasonics, Teltow, Germany), during which time the temperature of the solution increased to above ambient temperature but less than $50^{\circ} \mathrm{C}$. This extraction protocol simulates the baseline extraction protocol for the LMC. After ultrasonication, the mixture was allowed to stand for $10 \mathrm{~min}$, and the cloudy supernatant that had formed above the sedimented JSC Mars-1 was decanted into $1.5 \mathrm{~mL}$ centrifuge tubes. To minimize loss of extracted pyrene by absorption to surfaces of the hydrophobic polymer centrifuge tubes, the tubes were first blocked with $0.5 \%$ $(\mathrm{w} / \mathrm{v})$ BSA in $100 \mathrm{mM}$ HEPES pH 7.4 (a method commonly used in immunoassays). The supernatants were clarified by centrifugation at $17,000 \mathrm{~g}$ for $10 \mathrm{~min}$ and stored at $2-8^{\circ} \mathrm{C}$ in glass vials until tested in the immunoassay.

\subsection{Enzyme-linked immunosorbent assay (ELISA) procedure}

Immunoassay function was assessed in an inhibition ELISA implemented in a microwell plate format designed to mimic the baseline LMC flight immunoassay format. MicroWell plates (Nunc MaxiSorp flat-bottom 96 well; Roskilde, Denmark) were coated with benzo[a]pyrene-BSA conjugate at a concentration of $1 \mu \mathrm{g} / \mathrm{mL}$ in $0.05 \mathrm{M}$ carbonate buffer ( $\mathrm{pH} 9.6$, $100 \mu \mathrm{L} /$ well) and covered with adhesive plate-sealing film (Fisher Scientific, Loughborough, UK) to prevent evaporation. After $1 \mathrm{~h}$ incubation at a temperature of $37^{\circ} \mathrm{C}$, plates were washed with PBS-Tween [0.01 $M$ phosphate buffer, $0.0027 \mathrm{M}$ potassium chloride and $0.137 \mathrm{M}$ sodium chloride, $\mathrm{pH} 7.4$ with $0.05 \%(\mathrm{w} / \mathrm{v})$ Tween 20] and rinsed with water using an automatic plate washer (BW50 plate washer, Biohit, Helsinki, Finland). Uncoated binding sites on the plate surface were blocked with blocking buffer $[0.5 \%(\mathrm{w} / \mathrm{v})$ Cohn fraction V BSA in $100 \mathrm{mM}$ HEPES pH 7.4, $200 \mu \mathrm{L} /$ well] for $1 \mathrm{~h}$ at $37^{\circ} \mathrm{C}$. Plates were then washed as before.

To construct a standard curve, pyrene antigen was dissolved in LMC solvent $[20 / 80 \%(\mathrm{v} / \mathrm{v})$ methanol/water with $1.5 \mathrm{~g} / \mathrm{L}$ polysorbate 80$]$ at a concentration of $12,500 \mathrm{ng} / \mathrm{mL}$ and diluted with LMC solvent to obtain standard solutions in the concentration range $12,500-6.1 \mathrm{ng} / \mathrm{mL}$. Where standard curves were run in the presence of perchlorate salts, calcium perchlorate tetrahydrate or magnesium perchlorate hexahydrate were dissolved directly in the LMC solvent used to dilute pyrene for the standard curve at a concentration of $16.7 \mathrm{mM}$. This is equivalent to the expected perchlorate concentration if a $1 \mathrm{~g}$ sample of regolith containing $1 \mathrm{wt} \%$ perchlorate anion is extracted with $3 \mathrm{~mL}$ of LMC solvent assuming $100 \%$ extraction efficiency of perchlorate.

In the LMC flight format immunoassay, the liquid extract from the martian sample is pumped via microfluidic channels into a chamber containing a freeze-dried cocktail of immunoassay reagents including the antibodies. The solution is incubated in this chamber for approximately $1 \mathrm{~h}$ before it is transferred into a chamber containing an immobilized microarray of protein conjugates of target molecules. To mimic this in the microwell plate, immunoassay reagents were diluted at $2 \times$ the final desired concentration: mouse anti-benzo[a]pyrene clone 22F12 $(0.5 \mu \mathrm{g} / \mathrm{mL})$, goat anti-mouse IgG Fc biotin $(0.2 \mu \mathrm{g} / \mathrm{mL})$, and streptavidinHRP $(0.15 \mu \mathrm{g} / \mathrm{mL})$ (figures in parenthesis are $2 \times$ the final desired concentration) in LMC solvent $[20 / 80 \%(\mathrm{v} / \mathrm{v})$ methanol/water with $1.5 \mathrm{~g} / \mathrm{L}$ polysorbate 80$]$, along with the other immunoassay reagents in the freeze-dried cocktail also at $2 \times$ the final required concentration $[2 \times$ final concentration $=$ $20 \%(\mathrm{w} / \mathrm{v})$ sucrose, $100 \mu \mathrm{g} / \mathrm{mL}$ Allura Red AC dye-as a visible dye tracer, $1 \mathrm{mg} / \mathrm{mL}$ Cohn fraction V BSA, $0.02 \%(\mathrm{w} /$ v) thimerosal, $200 \mathrm{mM}$ HEPES $\mathrm{pH}$ 7.4] and then added 1:1 with the extracted sample or standard under test.

Antibodies and other freeze-dried cocktail reagents were incubated together with the sample/standard under test for 
$1 \mathrm{~h}$ at room temperature before transfer to the coated and blocked microwell plate (100 $\mu \mathrm{L} /$ well), where a further $1 \mathrm{~h}$ incubation took place. Plates were then washed as described previously. The baseline LMC flight immunoassay format will have a fluorescent readout, but for the purposes of the microwell immunoassay enzyme (HRP), labeled antibodies were used to generate a colorimetric signal through reaction with a substrate. Substrate solution was prepared by dis-

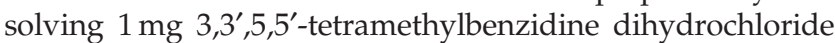
in $0.05 \mathrm{M}$ phosphate citrate buffer containing $0.03 \%$ sodium perborate $\mathrm{pH}$ 5.0. Substrate solution was added $(100 \mu \mathrm{L} /$ well), and color development was allowed to occur for approximately $10 \mathrm{~min}$. The enzyme reaction was stopped with $1 \mathrm{M}$ sulfuric acid (50 $\mu \mathrm{L} /$ well), and the absorbance was read at a wavelength of $450 \mathrm{~nm}$ with a microwell plate spectrometer (Varioskan Flash, Thermo Scientific, Leicestershire, $\mathrm{UK}$ ). Immunoassay $\mathrm{IC}_{50}$ (concentration of pyrene that produced a $50 \%$ drop in immunoassay signal intensity) was calculated by fitting data to a four-parameter binding equation (Findlay and Dillard, 2007).

\section{Experimental and Results}

\subsection{Experiment design}

Pyrene was selected as an LMC-relevant model target for this study since pyrene is an abiotically produced organic molecule, which has previously been detected in meteorites (Sephton and Botta, 2005) and may have been delivered to the martian surface by meteoritic infall. More importantly, an antibody that recognizes pyrene was readily available at the time of this work and has previously been demonstrated in LMC-relevant immunoassay formats. The monoclonal antibody clone 22F12 used in the pyrene immunoassay was originally raised against benzo[a]pyrene but had also been shown to cross react with pyrene and a range of other polyaromatic hydrocarbons (Matschulat et al., 2005). While the sensitivity of this immunoassay is appropriate for the current study, more sensitive immunoassays are in development for use in the flight instrument.

A martian regolith simulant developed by the Johnson Space Center (Houston, USA) (Allen et al., 1997), JSC Mars-1 was selected for use in this study because it is commonly used in studies where a Mars analogue is required (Court et al., 2010; Gough et al., 2010; Kral et al., 2011).

Perchlorate salts were spiked into JSC Mars-1 at $1 \mathrm{wt} \%$ perchlorate anion, as this concentration is of the same order of magnitude as the levels detected by the Phoenix lander. Hydrated calcium and magnesium salts were selected for use in this study because Hecht et al. (2009) concluded that the perchlorates detected by Phoenix were most likely to be calcium or magnesium salts, and given the evidence that water was present at the Phoenix landing site (Stoker et al., 2010), we felt it was reasonable to assume that the perchlorate salts are in hydrated form.

\subsection{Inhibition immunoassays with pyrene standards spiked with perchlorates}

To confirm that Mars-relevant concentrations of perchlorate salts in solution do not adversely interact with immunoassay reagents, inhibition immunoassays were performed with pyrene standards prepared in LMC solvent spiked with perchlorate salts. The standard curves generated (Fig. 1) confirmed that the immunoassay functioned in the presence of Mars-relevant levels of both calcium and magnesium perchlorate; as the concentration of pyrene in the standard was increased, inhibition of assay signal also increased. However, there were subtle differences between certain samples. Specifically the magnesium perchlorate-spiked standards showed a consistent reduction in assay signal for all pyrene concentrations compared to the calcium perchloratespiked standards and to the control standard without perchlorate. Calculated $\mathrm{IC}_{50}$ values for the immunoassay were $330 \mathrm{ng} / \mathrm{mL}$ pyrene for standards with no added perchlorate, $360 \mathrm{ng} / \mathrm{mL}$ pyrene for standards spiked with $16 \mathrm{mM}$ calcium perchlorate, and $160 \mathrm{ng} / \mathrm{mL}$ pyrene for standards spiked with $16 \mathrm{mM}$ magnesium perchlorate, again showing a change for the magnesium perchlorate-containing pyrene standards compared to the other standards.

To confirm that no significant $\mathrm{pH}$ change occurred in the immunoassay due to the dissolved perchlorate salts, the $\mathrm{pH}$ of the solution of perchlorate salts dissolved into the LMC extraction solvent containing the freeze-dried cocktail of immunoassay reagents and antibodies (which included a final concentration of $100 \mathrm{mM}$ HEPES buffer) was measured and found to be in the range of $\mathrm{pH}$ 7.0-7.5.

\subsection{Inhibition immunoassays with extracts from pyrene- and perchlorate-spiked samples}

To confirm that the presence of perchlorate salts in a dry sample did not affect the overall LMC analysis, including the

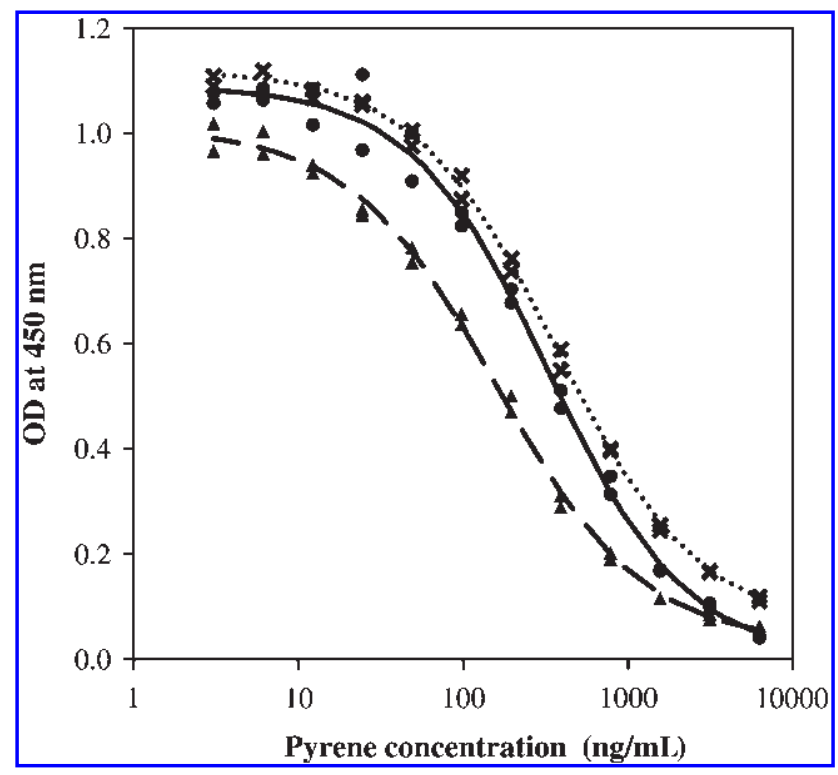

FIG. 1. Pyrene inhibition ELISA run in $20 / 80 \%$ (v/v) methanol/water with $1.5 \mathrm{~g} / \mathrm{L}$ polysorbate 80 and perchlorate salts. Each data point represents a single replicate for immunoassays run with $(\bullet)$ no perchlorate, $(\boldsymbol{x}) 16.7 \mathrm{mM}$ calcium perchlorate, and ( $\mathbf{\Delta}) 16.7 \mathrm{mM}$ magnesium perchlorate. Plotted immunoassay curves are the fit to a four-parameter binding equation for immunoassays run with (solid line) no perchlorate, (dotted line) $16.7 \mathrm{mM}$ calcium perchlorate, and (dashed line) $16.7 \mathrm{mM}$ magnesium perchlorate. OD, optical density. 
extraction step, a martian regolith simulant JSC Mars-1 was spiked with pyrene and perchlorate and extracted with LMC solvent; the extracts were analyzed by immunoassay. Note that no attempt was made to determine the efficiency of perchlorate dissolution into the LMC extraction solvent. Samples of JSC Mars-1 were first spiked with $500 \mu \mathrm{g} / \mathrm{g}$ or $0 \mu \mathrm{g} / \mathrm{g}$ pyrene; then dry calcium perchlorate or magnesium perchlorate was added to a level of $1 \mathrm{wt} \%$ of perchlorate anion. Control samples containing no perchlorate were also prepared and extracted.

While carrying out the extractions, we observed that, after centrifugation, extracts from $500 \mu \mathrm{g} / \mathrm{g}$ and $0 \mu \mathrm{g} / \mathrm{g}$ pyrenespiked JSC Mars-1 samples with $1 \mathrm{wt} \%$ calcium or magnesium perchlorate were colorless liquids, whereas extracts from $500 \mu \mathrm{g} / \mathrm{g}$ and $0 \mu \mathrm{g} / \mathrm{g}$ pyrene-spiked JSC Mars- 1 samples with no added perchlorate were yellow in color (Fig. 2). The $\mathrm{pH}$ of each of the extracts was measured; all extracts had a similar $\mathrm{pH}$ of approximately 7.0. A proportion of each extract was set aside for analysis by ELISA. Further experimentation on the remaining volumes of extracts revealed that addition of $16 \mathrm{mM}$ perchlorate salts or magnesium chloride salts directly to the yellow extract resulted in precipitation or aggregation of a yellow colloid-like suspension that could be removed by centrifugation to leave a colorless liquid.

Figure 3 shows the immunoassay curves produced by running dilutions of extracts from spiked samples in the immunoassay: extracts from six samples comprising combinations of pyrene spike level (0 or $500 \mu \mathrm{g} / \mathrm{g}$ ) and perchlorate spike (no perchlorate or $1 \mathrm{wt} \%$ calcium perchlorate or $1 \mathrm{wt} \%$ magnesium perchlorate).

For all extracts from samples spiked with perchlorate (both calcium and magnesium), the immunoassay performed as expected in that extracts from pyrene-spiked samples produced inhibition of the assay signal (Fig. 3a); for samples without pyrene, no inhibition of the assay signal occurred (Fig. 3b). For all extracts from samples without perchlorate, however, anomalous behavior was observed. For the extract from the sample spiked with $500 \mu \mathrm{g} / \mathrm{g}$ pyrene, at low extract concentration, assay signal was inhibited, but at higher extract

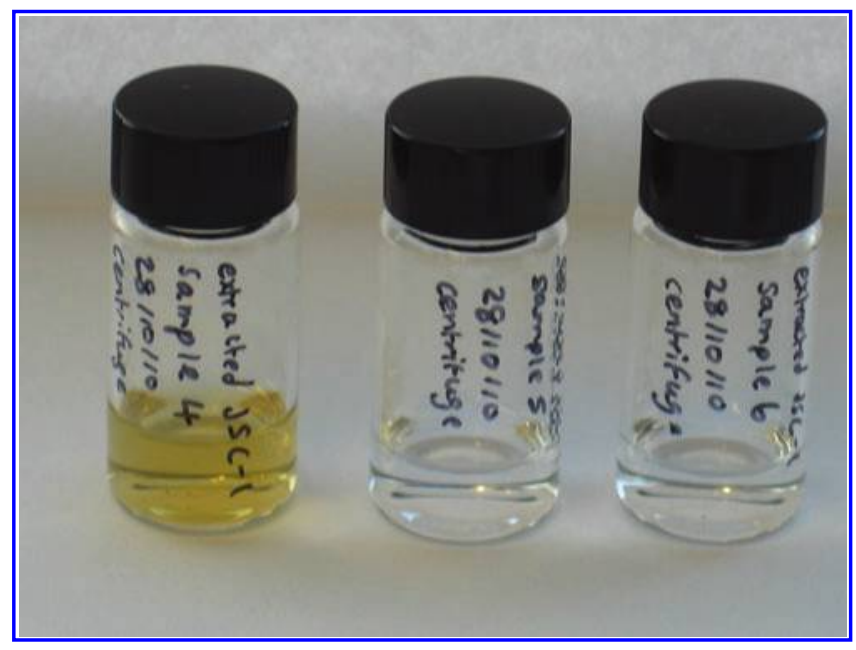

FIG. 2. Photograph of extracts from pyrene-spiked JSC Mars-1 samples. JSC Mars-1 spiked with $500 \mu \mathrm{g} / \mathrm{g}$ pyrene and (from left to right) spiked with no perchlorate, $1 \mathrm{wt} \%$ calcium perchlorate, and $1 \mathrm{wt} \%$ magnesium perchlorate prior to extraction. concentration $(>6 \%)$, a significant reduction in the level of assay signal inhibition was seen such that at an extract concentration of $50 \%$ no inhibition of assay signal occurred (Fig. 3a). For the extract from JSC Mars-1 without pyrene spike, at low extract concentration no inhibition of assay signal was observed, but at higher extract concentration $(>6 \%)$, a gradual reduction in the level of assay signal was seen such that at an

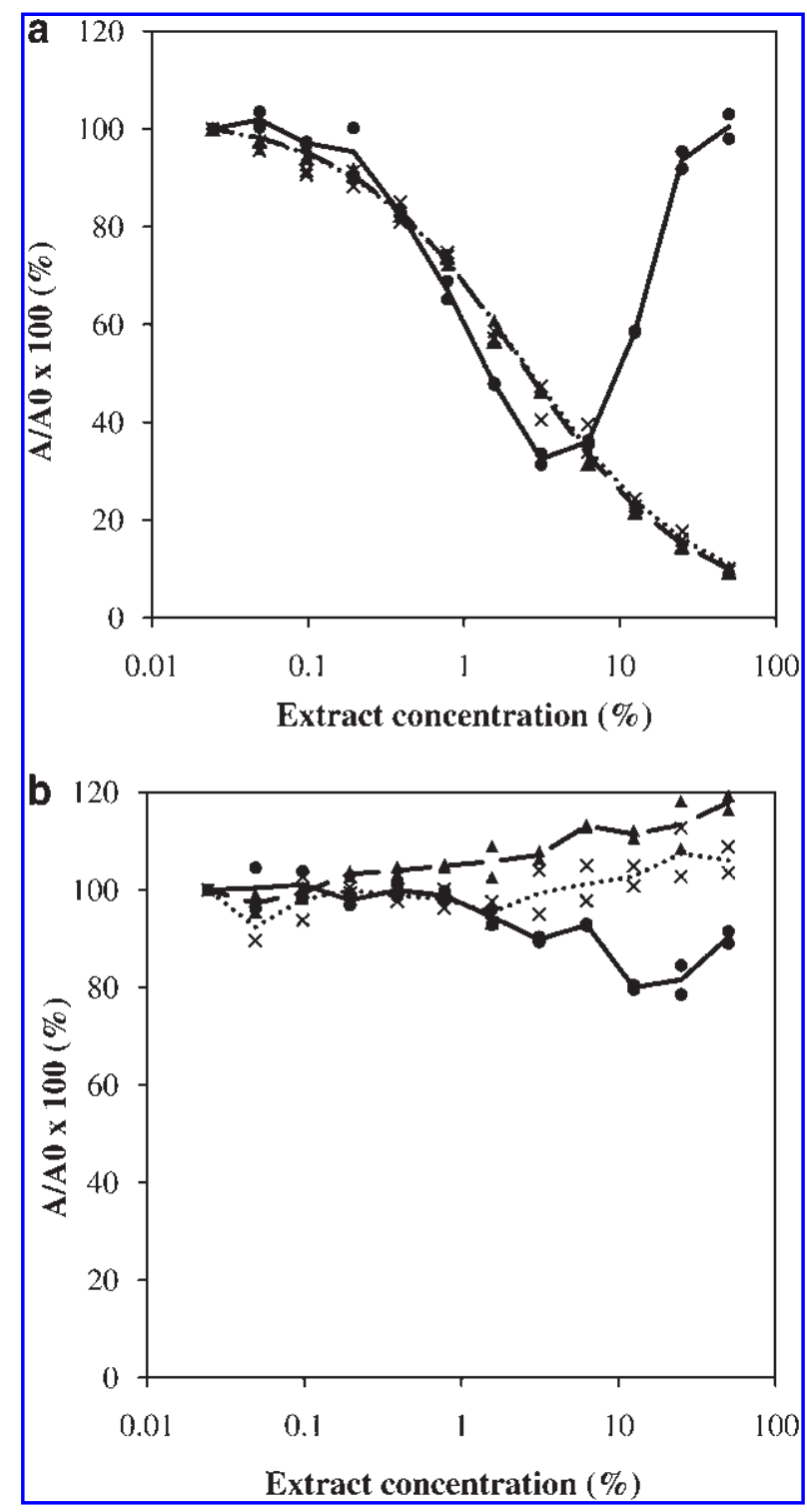

FIG. 3. Pyrene inhibition ELISA run with extracts diluted in $20 / 80 \%$ (v/v) methanol/water with $1.5 \mathrm{~g} / \mathrm{L}$ polysorbate 80 . Extractions performed on JSC Mars-1 spiked with (a) $500 \mu \mathrm{g} / \mathrm{g}$ pyrene (b) $0 \mu \mathrm{g} / \mathrm{g}$ pyrene. In each graph, (•) no perchlorate, (x) 1 wt \% calcium perchlorate, and ( $\Delta$ ) 1\% wt \% magnesium perchlorate. Each data point represents a single replicate. OD values (A) were normalized against maximum binding signal with $0 \%$ extract $(\mathrm{A} 0)$. Plotted immunoassay curves are the fit to a four-parameter binding equation; or, where such a fit was deemed inappropriate, curves to show trends in the data were drawn by using linear interpolation between the mean normalized OD values. (Solid line) no perchlorate, (dotted line) $1 \mathrm{wt} \%$ calcium perchlorate, and (dashed line) $1 \mathrm{wt} \%$ magnesium perchlorate. 
extract concentration of $50 \%$ an inhibition of assay signal of approximately $15 \%$ occurred (Fig. $3 b$ ). The $\mathrm{IC}_{50}$ values were calculated from the immunoassay data for extracts from the samples spiked with both perchlorate and pyrene, as described in the Materials and Methods section, which resulted in values of $2.4 \%$ extract concentration for the calcium perchlorate-spiked sample and $2.3 \%$ extract concentration for the magnesium perchlorate-spiked sample. The immunoassay data for extracts from the sample spiked with pyrene but with no perchlorate was not appropriate to fit to a four-parameter binding model. Nevertheless, we estimated the $\mathrm{IC}_{50}$ value by using only values of extract concentration below 6\%, which resulted in an $\mathrm{IC}_{50}$ value of $1.0 \%$ extract concentration.

\section{Discussion}

\subsection{Inhibition immunoassays with pyrene standards spiked with perchlorates}

Assay signal (optical density) in Fig. 1 is inhibited with pyrene standards in the presence of both $16 \mathrm{mM}$ calcium perchlorate and $16 \mathrm{mM}$ magnesium perchlorate, which shows that the pyrene inhibition immunoassay functioned in the presence of Mars-relevant concentrations of perchlorates. This was not unexpected given two considerations, namely, that (a) under low-temperature conditions perchlorate salts are expected to be non-oxidizing and (b) the measured $\mathrm{pH}$ and calculated ionic strength conditions caused by the presence of perchlorates in the present study are within the normal range for an immunoassay. For $\mathrm{pH}$, the inclusion of $100 \mathrm{mM}$ buffer salts in the LMC immunoassay allows a degree of control of perchlorate-induced $\mathrm{pH}$ changes, while for ionic strength, the addition of $16 \mathrm{mM}$ level of perchlorate salts only results in a small change in ionic strength.

Addition of $16 \mathrm{mM}$ calcium perchlorate to pyrene standards had little impact on immunoassay binding signal or $\mathrm{IC}_{50}$ compared to the control immunoassay run with pyrene standards without added perchlorate, whereas addition of $16 \mathrm{mM}$ magnesium perchlorate to the pyrene standards reduced immunoassay signal and increased the sensitivity with $\mathrm{IC}_{50}$ reduced by approximately $50 \%$. The reason for this difference is uncertain, although it is unlikely to be related to the perchlorate anions, since we assume that both the calcium and magnesium salts will be fully dissociated in solution and therefore present at the same concentration in both sets of perchlorate-containing pyrene standards. Magnesium may increase the affinity of the antibody for the free antigen but not for the conjugate, which thus diminishes the signal and consequently increases the sensitivity of the inhibitory assay. This could be studied in further work by assessing the effect of other magnesium salts.

Given that the aim is for the LMC to perform a threshold measurement, that is, to determine whether a target molecule is present at a concentration above the instrument detection limit rather than to attempt to quantify target molecules, changes of this magnitude are unlikely to have a significant impact on the ability of the LMC to function successfully.

\subsection{Inhibition immunoassays with extracts from pyrene- and perchlorate-spiked samples}

Immunoassays with extracts from JSC Mars-1 sample spiked with $500 \mu \mathrm{g} / \mathrm{g}$ pyrene and $1 \mathrm{wt} \%$ perchlorate (Fig. 3a) all showed decreasing immunoassay signal as extract concentration was increased, which implies that pyrene was extracted from the spiked JSC Mars-1 and detected in the immunoassay. This conclusion is further supported by examination of the immunoassay curves produced with extracts from JSC Mars-1 spiked with $0 \mu \mathrm{g} / \mathrm{g}$ pyrene and $1 \mathrm{wt} \%$ perchlorate (Fig. 3b), where no reduction in immunoassay signal was observed with increasing extract concentration, which suggests that the reduction in immunoassay signal produced with extracts from $500 \mu \mathrm{g} / \mathrm{g}$ pyrene and $1 \mathrm{wt} \%$ perchlorate-spiked JSC Mars-1 was not caused by either the perchlorate salts or by an interfering substance extracted from the JSC Mars-1 simulant regolith. Therefore, these results indicate that the presence of Mars-relevant levels of perchlorate salts in a well-studied Mars analog sample matrix had no significant effect on LMC-representative combined sample extraction and inhibition immunoassay detection of a representative small organic molecule LMC target (pyrene).

The immunoassay curve produced with extract from JSC Mars-1 spiked with $500 \mu \mathrm{g} / \mathrm{g}$ pyrene but no perchlorate (Fig. 3a) is significantly, and unexpectedly, different from all the other relevant immunoassay curves presented in Fig. 1 and Fig. 3a. In addition to this, the immunoassay curve produced with the extract from the unspiked sample (no pyrene and no perchlorate) (Fig. 3b) showed a gradual reduction in binding signal at high extract concentrations, which was not observed with extracts spiked with perchlorates (no pyrene). The extract concentration over which this reduction in signal was observed was the same as the concentration that produced anomalous results for pyrene-spiked samples (no perchlorates) (Fig. 3a). This observation suggests a common underlying effect. We noted that the anomalous behavior correlated with the yellow coloration of the extracts that only occurred from samples where no perchlorate was added (Fig. 2). We therefore infer that the substance responsible for the yellow color of the extract, from samples that had no perchlorate, caused the anomalous changes in immunoassay signal intensity. Since the final immunoassay readout of the HRP enzyme label was carried out after the yellow-colored extract was washed away, any optical absorption due to the yellow coloration can be discounted. Therefore, we conclude that the species producing the yellow color interacted with the immunoassay reagents by an as yet unidentified mechanism. To better understand the absence of the yellow coloration in extracts from the perchlorate-containing samples, further investigations were carried out, and we observed that when perchlorate or chloride salts were directly added to a yellowcolored extract, precipitation of a fine yellow solid occurred. This yellow solid could be removed by centrifugation to give a colorless extract. Therefore, in extracts from JSC Mars- 1 spiked with 1 wt \% perchlorate, we suspect that the species producing the yellow color was precipitated due to the presence of the perchlorate salts and removed during clarification of the extracts by centrifugation, which resulted in colorless extracts. The mechanism for this observation is unknown, but it is unlikely to be $\mathrm{pH}$ related given that the $\mathrm{pH}$ of both the yellow and colorless extracts were similar to one another. We have not performed studies to date with other Mars-relevant salts to confirm whether this observation is perchlorate salt specific or a more general effect due to presence of salts.

In addition to the anomalous changes in binding signal, the slopes of the curves produced with the three extracts 
from pyrene-spiked JSC Mars-1 with and without perchlorate salts (Fig. 3a) also differs in that the $\mathrm{IC}_{50}$ value for the extract from a sample spiked with pyrene but no perchlorate was $50 \%$ lower than values for extracts from samples spiked with pyrene and calcium perchlorate or magnesium perchlorate. This observation could be a result of changes in the immunoassay or changes in the extraction, or both, and it is not possible to determine which from this study. One possibility is that some pyrene may have been lost from the extract during the precipitation of the yellow solid in extracts from perchlorate-spiked samples but retained in the extracts from samples with no perchlorate, where no precipitation of the yellow solid occurred.

These results highlight that there is a difference between performing LMC extractions and immunoassays with JSC Mars-1 spiked with and without perchlorate; the addition of perchlorate salts prevented a component of the JSC Mars-1 from interfering with the immunoassay but also doubled immunoassay $\mathrm{IC}_{50}$. This observation will be subject to further study to clarify any broader implications.

While the main objective of this study was met, that is, to demonstrate that immunoassays can be performed in the presence of martian levels of perchlorate, including an LMCrepresentative extraction step, an unexpected sample matrix effect was observed in the absence of perchlorate. This warrants further investigation, but JSC Mars-1 is only representative of one class of a diverse range of possible sample matrixes that may be encountered on Mars. Therefore, we do not anticipate that this sample matrix effect will be present in all cases.

Future work will be carried out in microarray format utilizing fluorescent labels to clarify whether the observed changes in immunoassay performance are related to the HRP enzyme label or are true changes in antibody affinity. This may result in the effects observed with the yellow-colored extracts becoming negligible. The flight instrument will not incorporate a centrifugation step; rather, the martian extract will be filtered through a $0.5 \mu \mathrm{m}$ pore-size filter prior to introduction into the microfluidic channel. Thus, it is important to understand the implications of the findings of this study in a more flight-representative format.

The LMC instrument will include up to 25 immunoassays; therefore, further work is required to determine whether other LMC-relevant immunoassays behave in a similar manner to the pyrene immunoassay in the presence of perchlorate salts. A selection of different sample matrixes representative of the types of sample that may be encountered on Mars will also be tested to determine whether the sample matrix type is significant. In addition, it should be taken into account that a spiked sample may not effectively mimic a real sample where organic molecules may be incorporated differently into the matrix; therefore, testing with natural samples spiked with Mars-relevant levels of perchlorate will be carried out. Ideally, we would perform trials with natural samples with naturally occurring perchlorate salts, but to our knowledge there are no terrestrial samples available with Mars-relevant levels of perchlorates.

\section{Conclusion}

An immunoassay to detect pyrene, an LMC-relevant target, was implemented in an LMC-representative immu- noassay format and initially tested in the presence of perchlorate salts. No significant change in immunoassay function was observed in the presence of Mars-relevant levels of perchlorate salts when directly added to an immunoassay. This immunoassay was then used to confirm that pyrene could be extracted from a spiked martian regolith simulant with an LMC-relevant low-temperature extraction in the presence of perchlorates, and detected by immunoassay. As a result, the immunoassay was shown to function in the liquid extract and to detect extracted pyrene. For the same model sample matrix in the absence of perchlorate salts, we observed anomalous effects; immunoassays performed in the extract showed unexpected assay signals that coincided with a yellow coloration of the extracts. This unexpected observation is being studied further. Therefore, assuming that the immunoassay and simulant regolith tested are representative of all immunoassays and sample matrixes to be encountered, we conclude that both LMC immunoassay detection and the LMC low-temperature aqueous extraction with ultrasonication are not negatively influenced by the presence of perchlorate salts in martian samples at levels similar to those detected at the NASA Phoenix landing site. These results increase confidence in the LMC's capability to detect small organic molecules on Mars in perchlorate-rich samples and extend the work of Parro et al. The testing of further combinations of immunoassay and sample matrixes will be performed to validate the assumption that the pyrene immunoassay and JSC Mars-1 material are broadly representative and will include testing within a multiplexed microarray immunoassay format.

\section{Acknowledgments}

This work has been funded by the Science and Technology Facilities Council, UK. We thank Prof. Dr. D. Knopp of Technical University of Munich for the kind donation of antibenzo[ $a]$ pyrene antibody clone 22F12 and for guidance in its use. The authors would also like to thank LMC Consortium members, both past and present, especially those based at Cranfield University who have indirectly contributed to the work presented here.

\section{Author Disclosure Statement}

No competing financial interests exist.

\section{Abbreviations}

BSA, bovine serum albumin; ELISA, enzyme-linked immunosorbent assay; GC-MS, gas chromatograph-mass spectrometer; HRP, horseradish peroxidase; LMC, Life Marker Chip; OD, optical density; TEGA, Thermal and Evolved Gas Analyser.

\section{References}

Allen, C.C., Morris, R.V., Lindstrom, D.J., Lindstrom, M.M., and Lockwood, J.P. (1997) JSC Mars-1: martian regolith simulant [abstract 1797]. In 28 $8^{\text {th }}$ Lunar and Planetary Science Conference, Lunar and Planetary Institute, Houston.

Anderson, D.M., Biemann, K., Orgel, L.E., Oro, J., Owen, T., Shulman, G.P., Toulmin, P., and Urey, H.C. (1972) Mass spectrometric analysis of organic compounds, water and 
volatile constituents in the atmosphere and surface of Mars: the Viking Mars Lander. Icarus 16:111-138.

Court, R.W., Baki, A.O., Sims, M.R., Cullen, D., and Sephton, M.A., (2010) Novel solvent systems for in situ extraterrestrial sample analysis. Planet Space Sci 58:1470-1474.

Eigenbrode, J., Glavin, D., Dworkin, J., Conrad, P., and Mahaffy, P. (2011) Thermochemolysis-a new sample preparation approach for the detection of organic components of complex macromolecules in Mars rocks via gas chromatography mass spectrometry in SAM on MSL [abstract 1460]. In $42^{\text {nd }}$ Lunar and Planetary Science Conference, Lunar and Planetary Institute, Houston.

ESA. (2011) The ExoMars Rover Instrument Suite. MOMA-Mars Organics Molecule Analyser. European Space Agency, Noordwijk, the Netherlands. Available online at http://sci.esa.int/ science-e $/$ www $/$ object $/$ index.cfm?fobjectid $=45103 \&$ fbodylo ngid $=2132$.

Evans-Nguyen, T., Becker, L., Doroshenko, V., and Cotter, R.J. (2008) Development of a low power, high mass range spectrometer for Mars surface analysis. Int J Mass Spectrom 278:170-177.

Findlay, J.W.A. and Dillard, R.F. (2007) Appropriate calibration curve fitting in ligand binding assays. AAPS J 9, doi:10.1208/ aapsj0902029.

Gough, R.V., Tolbert, M.A., McKay, C.P., and Toon, O.B. (2010) Methane adsorption on a martian soil analogue: an abiogenic explanation for methane variability in the martian atmosphere. Icarus 207:165-174.

Hecht, M.H., Kounaves, S.P., Quinn, R.C., West, S.J., Young, S.M.M., Ming, D.W., Catling, D.C., Clark, B.C., Boynton, W.V., Hoffman, J., DeFlores, L.P., Gospodinova, K., Kapit, J., and Smith, P.H. (2009) Detection of perchlorate and the soluble chemistry of martian soil at the Phoenix lander site. $\underline{S c i-}$ ence 325:64-67.

Hoffman, J.H., Chaney, R.C., and Hammack, H. (2008) Phoenix Mars mission-the Thermal Evolved Gas Analyzer. I Am Soc Mass Spectrom 19:1377-1383.

Houtkooper, J.M. and Schulze-Makuch, D. (2007) A possible biogenic origin for hydrogen peroxide on Mars: the Viking results reinterpreted. International Journal of Astrobiology 6:147-152.

Kerr, R.A. (2010) Growing prospects for life on Mars divide astrobiologists. Science 330:26-27.

Kounaves, S.P., Hecht, M.H., Kapit, J., Gospodinova, K., DeFlores, L., and Quinn, R.C. (2010) Wet chemistry experiments on the 2007 Pheonix Mars Scout Lander mission: data analysis and results. J Geophys Res 115, doi:10.1029/2009JE003424.

Kral, T.A., Altheide, T.S., Lueders, A.E., and Schuerger, A.C. (2011) Low pressure and dessication effects on methanogens: implications for life on Mars. Planet Space Sci 59:264-270.

Matschulat, D., Deng, A., Niessner, R., and Knopp, D. (2005) Development of a highly sensitive monoclonal antibody based ELISA for detection of benzo[a]pyrene in potable water. Analyst 130:1078-1086.

Navarro-González, R., Vargas, E., de la Rosa, J., Raga, A.C., and McKay, C.P. (2010) Reanalysis of the Viking results suggests perchlorate and organics at midlatitudes on Mars. J Geophys Res 115, doi:10.1029/2010JE003599.

Parnell, J., Cullen, D., Sims, M.R., Bowden, S., Cockell, C.S., Court, R., Ehrenfreund, P., Gaubert, F., Grant, W., Parro, V., Rohmer, M., Sephton, M., Stan-Lotter, H., Steele, A., Toporski, J., and Vago, J. (2007) Searching for life on Mars: selection of molecular targets for ESA's Aurora ExoMars mission. Astrobiology 7:578-604.

Parro, V., de Diego-Castilla, G., Rodrígues-Manfredi, J.A., Rivas, L.A., Blanco-López, Y., Sebastián, E., Romeral, J., Compositizo, C., Herrero, P.L., García-Marín, A., Moreno-Paz, M., García-Villadangos, M., Cruz-Gil, P., Peinado, V., MartínSoler, J., Pérez-Mercader, J., and Gómez-Elvira, J. (2011) SOLID3: a multiplex antibody microarray-based optical sensor instrument for in situ life detection in planetary exploration. Astrobiology 11:15-28.

Posthuma-Trumpie, G.A., Korf, J., and van Amerongen, A. (2009) Lateral flow (immuno)assay: its strengths, weaknesses, opportunities and threats. A literature survey. Anal Bioanal Chem 393:569-582.

Sephton, M.A. and Botta, O. (2005) Recognizing life in the Solar System: guidance from meteoritic organic matter. International Journal of Astrobiology 4:269-276.

Shriver, D.F. and Atkins, P.W. (1999) Inorganic Chemistry, $3^{\text {rd }}$ ed., Oxford University Press, Oxford.

Sims, M.R., Cullen, D.C., Bannistera, N.P., Grant, W.D., Henry, O., Jones, R., McKnight, D., Thompson, D.P., and Wilson, P.K. (2005) The Specific Molecular Identification of Life Experiment (SMILE). Planet Space Sci 53:781-791.

Stoker, C.R., Zent, A., Catling, D.C., Douglas, S., Marshall, J.R., Archer, D., Clark, B., Kounaves, S.P., Lemmon, M.T., Quinn, R., Renno, N., Smith, P.H., and Young, S.M.M. (2010) Habitability of the Phoenix landing site. J Geophys Res 115, doi:10.1029/2009JE003421.

ten Kate, I.L. (2010) Organics on Mars? Astrobiology 10:589-603. Vago, J.L. and Kminek, G. (2007) Putting together an exobiology mission: the ExoMars example. In Complete Course in Astrobiology, edited by G. Horneck and P. Rettberg, Wiley-VCH, Weinheim.
Address correspondence to: Catherine Rix Cranfield Health Vincent Building Cranfield University Cranfield Bedfordshire, MK43 OAL $U K$

E-mail: c.rix@cranfield.ac.uk

Submitted 6 April 2011 Accepted 15 August 2011 